
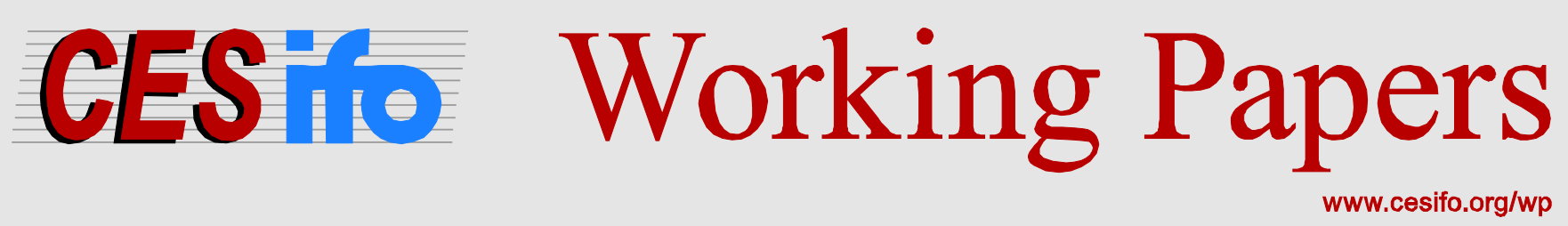

\title{
Financing and Funding Health Care: Optimal Policy and Political Implementability
}

\author{
Robert Nuscheler \\ Kerstin Roeder
}

CESIFO WORKING PAPER NO. 4893

CATEGORY 3: SOCIAL PROTECTION

JULY 2014

An electronic version of the paper may be downloaded

- from the SSRN website:

- from the RePEc website:

- from the CESifo website:

WWW.SSRN.com

Www.RePEc.org

www.CESifo-group.org/wp

\section{CESifo}




\title{
Financing and Funding Health Care: Optimal Policy and Political Implementability
}

\begin{abstract}
Health care financing and funding are usually analyzed in isolation. This paper combines the corresponding strands of the literature and thereby advances our understanding of the important interaction between them. We investigate the impact of three modes of health care financing, namely, optimal income taxation, proportional income taxation, and insurance premiums, on optimal provider payment and on the political implementability of optimal policies under majority voting. Considering a standard multi-task agency framework we show that optimal health care policies will generally differ across financing regimes when the health authority has redistributive concerns. We show that health care financing also has a bearing on the political implementability of optimal health care policies. Our results demonstrate that an isolated analysis of (optimal) provider payment rests on very strong assumptions regarding both the financing of health care and the redistributive preferences of the health authority.
\end{abstract}

JEL-Code: H240, I140, I180.

Keywords: health care financing, provider payment, service quality, cost containment, political economy.

Robert Nuscheler

University of Augsburg

Department of Economics

Universitätsstr. 16

Germany - 86150 Augsburg

robert.nuscheler@wiwi.uni-augsburg.de
Kerstin Roeder*

University of Munich

Department of Economics

Ludwigstr. 28

Germany - 80539 Munich

kerstin.roeder@Irz.uni-muenchen.de

*corresponding author

July 2, 2014 


\section{Introduction}

Health care funding traditionally receives great attention by health economists. The primary topic of interest is how health care providers should be paid and how the environment, e.g., competition and information, shapes the optimal reimbursement system (see Chalkley and Malcomson, 2000, for an overview). The question of how the required revenue to reimburse providers should be raised, that is, how health care financing should be organized, has received less attention. Financing frameworks typically assume a positive perspective and investigate how the politico economy equilibrium depends on voter heterogeneity (see, e.g., Epple and Romano, 1996, and Gouveia, 1997). Surprisingly, health care financing and funding are typically analyzed in isolation: research on provider payment ignores how health care is being financed and the financing literature neglects how the funds are being used. By simultaneously analyzing health care financing and funding our study fills this gap and thereby advances the understanding of the important health care financing and funding interaction.

We investigate the impact of three modes of health care financing, namely, optimal income taxation, proportional income taxation, and insurance premiums, on optimal provider payment and on the political implementability of optimal policies under majority voting. We consider a standard multi-task agency framework where the health care provider chooses the quality of care and cost reducing effort. More quality in health care is beneficial to patients and to the provider who is considered (partial) altruistic. While more quality increases treatment costs, cost reduction effort lowers them. Individuals differ along two dimensions, i.e., risk and income. Given this heterogeneity an allocation is assessed along three dimensions: quality, effort, and the distribution of income. If optimal income taxation is feasible - or redistributive concerns are absent - and if quality and effort are contractible, the first-best allocation can be implemented. When quality and effort are non-contractible, the health authority uses a linear cost-sharing arrangement to steer the provider's incentives to invest in quality and to exert effort. As the health authority has two margins but only one instrument the first-best allocation can no longer be implemented. The health authority then uses the cost-sharing parameter to optimally trade off the inefficiencies in quality and effort. If health care financing is through optimal income taxes, this tradeoff is not blurred by any redistributive consequences which the financing of health care provision might have.

This second-best allocation is then contrasted with allocations under alternative financing regimes, namely, proportional income taxes and insurance premiums. We call the resulting allocations third-best. With proportional income taxation, income is redistributed from highincome agents to low-income ones and from low-risk agents to high-risk ones. Depending on the distributional characteristics of risk and income the third-best policy may imply more cost-

sharing than the second-best policy and with it higher quality and less effort causing health care 
expenses to be higher. When health care financing is through insurance premiums the secondbest quality-effort tradeoff is affected if and only if insurance premiums involve some pooling. Then premiums redistribute income from low-risk agents to high-risk ones with the extent being governed by the degree of pooling. The comparison between the second-best and third-best allocations hinges on the distributional characteristic of risk and on the extent of pooling.

To complete the picture, we derive the allocations under majority voting and contrast them with the optimal policies for both proportional income taxes and insurance premiums. While the redistributive preferences of the health authority are governed by the distributional characteristics, the preferences of the median voter depend on individual heterogeneity. This implies that, only in knife-edge cases, can the optimal (third-best) policies be implemented as political equilibria. For the case of proportional income taxes the comparison of the two allocations depends on how the relative inequity between risk and income compares to the relative distributional characteristics between these two dimensions. For insurance premiums it is only the inequity in risk together with the extent of pooling and its relation to the distributional characteristic of risk that matters.

Finally, it should be noted that, rather remarkably, risk-rated premiums imply second-best optimal health care provision for both the third-best allocation and the political outcome. The reason being that risk-rated premiums preclude any form of redistribution. There is then no conflict in the electorate about how to shape the health care system and second-best health care provision results. From the normative end it does not pay off to distort the optimal policy away from the second-best as the associated efficiency losses are not compensated by redistributive gains. As the resulting income distribution may not be optimal the equilibrium allocation may not be second-best efficient. Our results demonstrate that studies on optimal provider payment that neglect health care financing rest on very strong assumptions regarding the redistributive motives of the health authority, or on health care financing.

This article relates to two strands of the health economics literature. First, provider payment. The papers of Chalkley and Malcomson (1998a; 1998b), Ma (1994), and, more recently, Eggleston (2005), and Kaarbøe and Siciliani (2011), show that mixed payment systems will generally be optimal. Whether quality incentives will be high powered or low powered depends on the respective environment. It may relate to the quality elasticity of demand as in the first three papers, or to the complementarity between the different dimensions under consideration (the latter two papers). We use a simplified version of their models enabling us to integrate health care financing. Second, the political economy of health care financing. Epple and Romano (1996), Gouveia (1997), Kifmann (2005), and Nuscheler and Roeder (2013) aim at explaining the existence of public health care, its size and its form of financing. In these models, health care is used to redistribute income. This literature, however, disregards how tax revenue is passed 
on to providers. Zeckhauser (1970) was the first to simultaneously analyze provider payment and health care financing. Ma and McGuire (1997) generalized this framework. These papers analyze optimal health insurance in an ex post moral hazard setting. We consider a multi-task agency set-up instead and investigate a much richer set of financing regimes. This allows us to analyze the important interaction between health care financing and funding in great detail and thereby to contribute to a rather slim financing and funding literature.

The remainder of the paper is organized as follows. Section 2 introduces the basic framework, followed by a normative analysis in Section 3 . Political outcomes are derived and compared to the respective normative allocations in Section 4 . Finally, Section 5 concludes.

\section{The Model}

\section{$2.1 \quad$ Individuals}

We consider a continuum of individuals who differ along the two most important dimensions when it comes to health care financing and health care provision, namely, income and risk. There are two income types $i=r, p$ (rich and poor) with income levels $y_{r}>y_{p}>0$. The probability of falling ill is denoted $\pi_{j}$ and assumes the value $\pi_{l} \in(0,1)$ for low-risk individuals and $\pi_{h} \in\left(\pi_{l}, 1\right)$ for high-risk individuals. Both income and risk are exogenously given. The two-dimensional heterogeneity gives rise to $i j$-types and we denote their share in the population $\theta_{i j} \in\left[0, \frac{1}{2}\right)$, where the upper bound is introduced for the sake of interest. 1 To ease notation we define $\theta_{i} \equiv \theta_{i l}+\theta_{i h}$ and $\theta_{j} \equiv \theta_{p j}+\theta_{r j}$. In the following, we assume $\theta_{p}>0.5$, that is, median income, $y_{p}$, is below average income, $\bar{y}=\theta_{p} y_{p}+\theta_{r} y_{r}$. In addition, the majority of individuals is exposed to a low health risk, $\theta_{l}>0.5$. This implies that median risk, $\pi_{l}$, is smaller than average risk, $\bar{\pi}=\theta_{l} \pi_{l}+\theta_{h} \pi_{h}$. Table 1 summarizes.

Table 1: Agent Heterogeneity.

\begin{tabular}{cc|c|c|c}
\hline \hline & & \multicolumn{2}{|c|}{ Income } & \\
& & $p$ & $r$ & $\Sigma$ \\
\hline \multirow{2}{*}{ Health Risk } & $l$ & $\theta_{p l}$ & $\theta_{r l}$ & $\theta_{l}>0.5$ \\
& $h$ & $\theta_{p h}$ & $\theta_{r h}$ & $\theta_{h}<0.5$ \\
\hline \hline & $\Sigma$ & $\theta_{p}>0.5$ & $\theta_{r}<0.5$ & 1 \\
\hline \hline
\end{tabular}

Sickness inflicts a disutility $L>0$ on individuals. Through the receipt of care these costs can be mitigated but not eliminated. We refer to this reduction as the benefit of treatment

\footnotetext{
${ }^{1}$ In our political game an $i j$-type with $\theta_{i j} \geq \frac{1}{2}$ could dictate the allocation.
} 
and denote it $b \in[0, L)$. There is one health care provider (HCP), e.g., a hospital, specialist, or general practitioner, who treats all patients in need of care. We summarize all provider activities that aim at increasing $b$ in a single, one-dimensional quality index $q \cdot{ }^{2}$ We let $b_{q}>0$ and $b_{q q} \leq 0$, that is, an improvement in health care quality increases the benefits from treatment but at a decreasing rate.

Health care is publicly financed. We distinguish between three financing regimes indexed $n \in\{T, t, p\}$. The government either uses individualized lump-sum transfers $T_{i j}$ (optimal income taxation, $n=T)$, proportional income taxes with tax rate $t \geq 0(n=t)$, or insurance premiums $(n=p)$ to generate the revenue required to reimburse the HCP. Insurance premiums can be either risk-based, pooled, or a be mixture of both. More precisely, the insurance premium is given by $\left[(1-\varphi) \pi_{j}+\varphi \bar{\pi}\right] p$, where $p$ is the price of health care and $\varphi \in[0,1]$ the extent of pooling.

In addition to the (expected) benefits from health care, agents derive utility from consumption of a numéraire commodity denoted $x$. Assuming quasi-linear preferences, the optimization problem of individual $i j$ can be written as $\mathrm{S}^{3}$

$$
\max _{x_{i}} U_{i j}=x_{i}+\pi_{j}[b(q)-L]
$$

where the budget constraint amounts to $x_{i}=y_{i}-T_{i j}, x_{i}=(1-t) y_{i}$, and $x_{i j}=y_{i}-\left[(1-\varphi) \pi_{j}+\varphi \bar{\pi}\right] p$ for financing regimes $T, t$, and $p$, respectively.

\subsection{The Health Care Provider}

When treating a patient the provider incurs treatment costs $K$. As health care always includes a random element, these costs are uncertain. From an ex ante perspective only expected treatment costs, $c=E(K)$, matter. Despite this uncertainty the HCP has an influence on costs and we suggest that $c$ is a function of the quality of care, $q$, and the provider's effort to keep treatment costs down, $e .4$ We let the expected treatment cost function $c(q, e)$ satisfy $c_{q}>0, c_{q q}>0$, $c_{e}<0, c_{e e} \geq 0$ and $c_{e q}=0$. Expected treatment costs, thus, increase with quality and do so at an increasing rate. More effort implies lower treatment costs in expectation but at a decreasing rate 5 The provider's effort to contain costs involves a disutility $v(e)$ per patient, where $v_{e}>0$ and $v_{e e}>0$. Finally, the HCP incurs a fixed cost $F$.

Total provider reimbursement is denoted $P$ and the actual payment is determined by a simple

\footnotetext{
${ }^{2}$ This assumption rules out multi-task quality issues when it comes to optimal provider payment (Chalkley and Malcomson, 1998a, 1998b, and Kaarbøe and Siciliani, 2011). As will become clear below we nevertheless face a multi-task problem.

${ }^{3} \mathrm{We}$ assume that patients passively accept the quality of treatment the HCP is willing to provide.

${ }^{4}$ Although we are not concerned with multiple quality dimensions we have a multi-task agency problem: the HCP chooses quality and effort.

${ }^{5}$ The assumption that the cross derivative vanishes is made for analytical convenience.
} 
linear cost-sharing arrangement, $P=\gamma K+\tau$. The parameter $\gamma \in[0,1]$ is the extent to which the health authority (HA) is willing to share treatment costs. As an additional compensation the HCP may receive a lump-sum payment $\tau \in \mathbb{R}$ per patient treated. The expected reimbursement is given by

$$
E(P)=\gamma c(q, e)+\tau
$$

In addition to reimbursement the $\mathrm{HCP}$ derives utility from the patients' benefits generated through treatment. This gives rise to the HCP's expected payoff:

$$
H(q, e)=\bar{\pi}[\alpha b(q)-c(q, e)-v(e)+E(P)]-F
$$

where $\bar{\pi}$ is the share of agents treated, that is, all individuals who are sick. The parameter $\alpha$ captures the HCP's degree of altruism towards patients' health benefits (see, e.g., Ellis and McGuire, 1986; Eggleston, 2005; Jack, 2005). For $\alpha=1$, the HCP is a 'perfect agent' and for $\alpha<1$ an imperfect one ${ }^{6}$

Substituting $E(P)$ in equation (3) by equation (2) we arrive at the HCP's optimization program

$$
\max _{q, e} H(q, e)=\bar{\pi}[\alpha b(q)-(1-\gamma) c(q, e)-v(e)+\tau]-F
$$

Our assumptions about the benefit function $b(q)$ and the cost functions $c(q, e)$ and $v(e)$ ensure that the HCP's problem is concave. The first-order conditions with respect to $q$ and $e$ amount to

$$
\begin{aligned}
& \frac{\partial H(q, e)}{\partial q}=0 \quad \Leftrightarrow \quad \alpha b_{q}-(1-\gamma) c_{q}=0 \\
& \frac{\partial H(q, e)}{\partial e}=0 \quad \Leftrightarrow \quad-(1-\gamma) c_{e}-v_{e}=0 .
\end{aligned}
$$

The above system of equations yields the optimal quality of health services $q(\gamma ; \alpha)$ and the optimal level of cost reducing effort $e(\gamma ; \alpha)$ as functions of the cost sharing parameter $\gamma$ and the degree of altruism $\alpha$. The optimal quality of health care services is determined such that the internalized marginal benefit of treatment (the first term of equation (5)) is equal to the marginal cost of providing quality (the second term). The optimal cost reducing effort is chosen to equalize the marginal benefit of lower treatment costs (the first term of equation (6)) with

\footnotetext{
${ }^{6}$ Note that even in the case of perfect agency, the individuals' well-being does not fully enter the HCP's utility function. The reason is that even though the HCP considers the patients' utility from treatment, it does not take into account the financial costs of service delivery, that is, the taxes or premiums that are borne by the patients (see also Jack, 2005). So, the HCP's and the patient's valuation of health care services may differ even if $\alpha=1$.
} 
the marginal disutility of effort (the second term).

The actual quality and effort levels the HCP is willing to provide depend on the contract he has. Specifically, it depends on the cost sharing component $\gamma$. With the help of Cramer's rule (see the Appendix A.2), we get

$$
\begin{aligned}
& q_{\gamma} \equiv \frac{\mathrm{d} q(\gamma ; \alpha)}{\mathrm{d} \gamma}=\frac{-c_{q}}{\alpha b_{q q}-(1-\gamma) c_{q q}}>0, \\
& e_{\gamma} \equiv \frac{\mathrm{d} e(\gamma ; \alpha)}{\mathrm{d} \gamma}=\frac{c_{e}}{(1-\gamma) c_{e e}+v_{e e}}<0
\end{aligned}
$$

The more cost-based the HCP's payment the larger the quality he is willing to supply and the smaller the effort he exerts to reduce cost. The first effect is due to the lower price the HCP has to pay for quality improvements if the cost-sharing parameter $\gamma$ increases. The less expensive the provision of quality the more the HCP is willing to offer. The second effect is a moral hazard effect. An increase in the share of reimbursed treatment costs undermines the HCP's incentives to contain costs. Additionally, $q(\gamma ; \alpha)$ positively depends on $\alpha$ : the more the HCP cares about the patients' health care benefits, the higher the quality of health care services it delivers.

\subsection{The Public Health Care Scheme}

The government or the health authority (who is the purchaser of health care services) faces two constraints, a participation constraint and a budget constraint. We assume throughout that the benefit from treatment is sufficiently large so that the HA always wants to contract with the HCP. As the HCP cannot be forced to provide health care services, the lump-sum transfer $\tau$ per patient and the cost-sharing parameter $\gamma$ must be chosen such that the HCP is willing to accept the contract. With a reservation utility of zero the participation constraint reads a: 7

$$
\bar{\pi}[\alpha \beta b(q)-(1-\gamma) c(q, e)-v(e)+\tau]-F \geq 0 .
$$

The parameter $\beta \in\{0,1\}$ allows us to distinguish between two scenarios. For $\beta=0$ the monetary part of the HCP's utility needs to be non-negative. In contrast, for $\beta=1$ the participation constraint is less demanding as the (partial) internalization of patients' health benefits allows for negative monetary payoffs. The functional form of $(9)$ and the parameters $\alpha$ and $\beta$ are common knowledge.

As already noted above, the expected health care expenses, $H C E=\bar{\pi}[\gamma c(q, e)+\tau]$, can either be financed by optimal income taxes, proportional income taxes, or insurance premiums. To balance the public budget, public revenues need to be equal to the expected health care

\footnotetext{
${ }^{7} \mathrm{~A}$ strictly positive reservation utility would simply add to the fixed costs of being active in the market.
} 
expenses. We have

$$
\begin{aligned}
\Sigma_{i j} \theta_{i j} T_{i j} & \equiv \bar{T}=H C E \\
t \Sigma_{i} \theta_{i} y_{i} & \equiv t \bar{y}=H C E \\
p \Sigma_{j} \theta_{j}\left[(1-\varphi) \pi_{j}+\varphi \bar{\pi}\right] & =p \bar{\pi}=H C E
\end{aligned}
$$

for regimes $T, t$, and $p$, respectively. In equilibrium the participation constraint will be binding so that we can solve equation (9) for $\tau$. Inserting $\tau$ into the budget constraints $(10)-(12)$ we obtain one condition for each regime that guarantees both, a balanced public budget and participation of the HCP:

$$
\begin{aligned}
\bar{T}(\gamma) & =\bar{\pi}[c(q, e)+v(e)-\alpha \beta b(q)]+F, \\
t(\gamma) & =\frac{\bar{\pi}}{\bar{y}}\left[c(q, e)+v(e)-\alpha \beta b(q)+\frac{F}{\bar{\pi}}\right], \\
p(\gamma) & =[c(q, e)+v(e)-\alpha \beta b(q)]+\frac{F}{\bar{\pi}} .
\end{aligned}
$$

Since $e=e(\gamma)$ and $q=q(\gamma)$, the budget balancing revenue depends on the cost sharing parameter $\gamma$ only indirectly, that is, via the influence cost sharing has on the HCP's optimally chosen quality and effort. With the help of the implicit function theorem and observing the first order conditions of the HCP, equations (5) and (6), we have

$$
\frac{\mathrm{d} \bar{T}(\gamma)}{\mathrm{d} \gamma}=\bar{y} \frac{\mathrm{d} t(\gamma)}{\mathrm{d} \gamma}=\bar{\pi} \frac{\mathrm{d} p(\gamma)}{\mathrm{d} \gamma}=\bar{\pi}\left[\left(c_{q}-\alpha \beta b_{q}\right) q_{\gamma}+\left(c_{e}+v_{e}\right) e_{\gamma}\right]>0
$$

A higher cost-sharing parameter requires higher public revenues. This is a direct consequence of the HCP's response to an increase in cost-sharing: more cost-sharing implies higher quality (7) and less cost reducing effort (8) both increasing expected health care spending and with it the revenues needed to balance the public budget.

\subsection{The Economic Equilibrium}

The following definition introduces the notion of economic equilibrium into our model economy.

\section{Definition 1 (Economic Equilibrium)}

An allocation $\left\{x_{p l}, x_{p h}, x_{r l}, x_{r h}, q, e\right\}$ with policy instruments $T_{i j} / t / p, \tau$ and $\gamma$ constitutes an equilibrium of the economy if the following conditions hold:

(i) the utility of all agents is maximized, i.e., the program given in equation (1) is solved,

(ii) the health care provider's participation constraint (9) is satisfied and its payoff is maxi- 
mized, i.e., the program given in equation (4) is solved, and

(iii) the government's budget constraint is balanced, i.e., for optimal income taxation equation (10) holds, for proportional income taxation equation (11) holds, and for risk-based premiums equation (12) holds.

In an economic equilibrium the utility level obtained by type- $i j$ agents can be expressed by their indirect utility function $V_{i j}^{n}(\gamma)$, where

$$
\begin{aligned}
V_{i j}^{T}(\gamma) & =y_{i}-T_{i j}(\gamma)+\pi_{j}[b(q(\gamma))-L], \\
V_{i j}^{t}(\gamma) & =(1-t(\gamma)) y_{i}+\pi_{j}[b(q(\gamma))-L] \\
V_{i j}^{p}(\gamma) & =y_{i}-\left[(1-\varphi) \pi_{j}+\varphi \bar{\pi}\right] p(\gamma)+\pi_{j}[b(q(\gamma))-L]
\end{aligned}
$$

The indirect utility function represents an individual's preferences over the cost sharing parameter, $\gamma$. Inspection of equations $(17)-(19)$ reveals that these preferences depend on the mode of health care financing. This already points to the different distributional properties of the three financing regimes. With individualized lump-sum transfers the government can perfectly redistribute between individuals so as to equalize their (marginal) utilities. With proportional income taxation, income can still be redistributed from rich to poor using health care as a vehicle (see, e.g., Gouveia, 1997). While any form of redistribution is ruled out when premiums are riskbased $(\varphi=0)$, partial pooling, $\varphi \in(0,1]$, implies redistribution from low-risk to high-risk agents with the extent of redistribution being increasing in $\varphi$. In Sections 3 and 4 we carefully analyze how these differences affect the allocations in normative and political economy environments.

\section{Optimal Financing and Funding}

In this section we study the optimal interaction between health care financing and funding considering four different allocations: first-best, second-best, and two third-best allocations. To determine the first-best allocation we consider quality and effort contractible and let optimal income taxation be feasible, that is, health care financing is through individualized lumpsum transfers. By maintaining the optimal income taxation assumption and introducing noncontractibility for both quality and effort we obtain the second-best allocation. This allocation is then compared to two third-best allocations where quality and effort are still considered noncontractible but alternative health care financing regimes are considered, namely, proportional income taxation and insurance premiums.

Throughout our analysis we assume a HA or government who may have redistributive concerns, that is, who may aim at redistributing to the double disadvantaged in society, that is, to high-risk low-income individuals. We incorporate redistributive considerations into the analysis 
by letting the HA's objective function be

$$
W=\sum_{i j} \theta_{i j} \Psi\left(U_{i j}\right)
$$

where $\Psi(\cdot)$ is a strictly increasing and weakly concave function of individual utility levels, i.e. $\Psi^{\prime}(\cdot)>0$ and $\Psi^{\prime \prime}(\cdot) \leq 0$. This formulation comprises a utilitarian HA as limiting case, $\Psi^{\prime \prime}(\cdot)=0$. Due to quasi-linearity every income distribution would then be optimal. In other words, all redistributive concerns of the $\mathrm{HA}$ are contained in $\Psi$. An immediate implication is that a necessary condition for a difference between the second-best and the two third-best allocations is the presence of redistributive concerns. In the following, we investigate how these concerns shape optimal health policy.

\subsection{Normative Benchmarks - Optimal Income Taxation}

\subsubsection{First-Best}

The HA maximizes 20 with respect to $T_{i j}, q$ and $e$ subject to the HCP's participation constraint (9) and the public budget constraint (10). The optimization problem is 8

$$
\begin{aligned}
\max _{T_{i j}, \tau, q, e} & W^{*}\left(T_{i j}, \tau, q, e\right)=\sum_{i j} \theta_{i j} \Psi\left(y_{i}-T_{i j}+\pi_{j}[b(q)-L]\right) \\
\text { s.t. } & \bar{\pi}[\alpha \beta b(q)-c(q, e)-v(e)+\tau]-F \geq 0 \quad \text { and } \quad \sum_{i j} \theta_{i j} T_{i j}=\bar{\pi} \tau .
\end{aligned}
$$

Inserting the participation constraint into the budget constraint and taking the derivative with respect to $T_{i j}, q$ and $e$ yields

$$
\begin{aligned}
& \frac{\partial W^{*}\left(T_{i j}, q, e\right)}{\partial T_{i j}}=-\theta_{i j} \Psi_{i j}^{\prime}+\mu \theta_{i j}=0 \quad \Leftrightarrow \quad \Psi_{i j}^{\prime}=\mu \quad \forall i j, \\
& \frac{\partial W^{*}\left(T_{i j}, q, e\right)}{\partial q}=-c_{q}+[1+\alpha \beta] b_{q}=0, \\
& \frac{\partial W^{*}\left(T_{i j}, q, e\right)}{\partial e}=c_{e}+v_{e}=0
\end{aligned}
$$

where $\mu$ is the Lagrangean multiplier on the budget constraint. The first condition states that individualized lump-sum transfers should be chosen such that marginal utilities are equalized. For $\Psi^{\prime \prime}<0$ this implies that, for a given risk type, high income individuals have to pay more taxes than low income agents: $T_{r j}>T_{p j}$. Similarly, for a given income type, low-risk agents

\footnotetext{
${ }^{8}$ Chalkley and Malcomson (1998b) argue that this welfare function includes double counting of the patient's health care benefits. Our results do not change qualitatively when the altruistic term is dropped from the HCP's participation constraint, that is, when $\beta=0$ (see also Kaarbøe and Siciliani, 2011).
} 
have to pay more taxes than high-risk agents as the former suffer the utility loss from illness with a higher probability than the latter: $T_{i l}>T_{i h}$. We get the following ordering of individual lump-sum taxes: $T_{r l}>\max \left\{T_{p l}, T_{r h}\right\} \geq \min \left\{T_{p l}, T_{r h}\right\}>T_{p h}$. Whether $T_{p l} \lessgtr T_{r h}$ depends on the relative inequity between income and risk. Without redistributive concerns, $\Psi^{\prime \prime}=0$, all $T_{i j}$ that balance the public budget would be optimal. Equation (23) states that first-best quality, $q^{*}$, should be expanded until the marginal benefit to patients, $b_{q}$, is equal to the marginal costs of providing health care services, $c_{q}-\alpha \beta b_{q}$. Obviously, the costs of expanding quality are lower when the participation constraint of the HCP includes an altruistic component $(\beta=1)$ as compared to a situation where the HCP has to break even in monetary terms $(\beta=0)$. As a result, the first-best efficient quality level is higher in the former case and the quality differential is increasing with $\alpha$. The first-best cost reduction effort, $e^{*}$, equates the marginal disutility of exerting effort, $v_{e}$, to the marginal expected treatment cost savings, $-c_{e}$; see equation (24).

\subsubsection{Second-Best}

The government can still impose optimal income taxes but both quality and effort are noncontractible 9 Using the reimbursement system the HA can steer the HCP's incentives to invest in quality and to contain costs. We focus on linear contracts as given in equation (2) that specify a lump-sum transfer per patient $\tau$ and a cost-sharing parameter $\gamma$.

Again, the HA maximizes the welfare function subject to the participation constraint (9) of the $\mathrm{HCP}$ and the public budget constraint (10). The HA, however, no longer optimizes over $T_{i j}, q$ and $e$, but over $T_{i j}$ and $\gamma$. In other words, she loses one degree of freedom as compared to the first-best problem. In determining the optimal cost-sharing parameter the HA has to consider the HCP's optimal quality and effort responses to cost sharing arrangements as given by equations (5) and (6). Specifically, the optimization problem is given by

$$
\begin{array}{ll}
\max _{T_{i j}, \gamma} & W^{T}\left(T_{i j}, \gamma\right)=\sum_{i j} \theta_{i j} \Psi\left(y_{i}-T_{i j}+\pi_{j}[b(q)-L]\right) \\
\text { s.t. } & \text { (5), (6) and 13. }
\end{array}
$$

After some rearrangements, the first order conditions with respect to $T_{i j}$ and $\gamma$ can be written

\footnotetext{
${ }^{9}$ While this assumption is rather plausible for cost reducing effort it is at odds with the increasing importance of pay-for-performance schemes. Nevertheless, the assumption can be justified on a number of grounds. First, the assumption is fairly standard in the earlier literature on provider payment (see, e.g., Chalkley and Malcomson, 1998a; 1998b). Second, even the more recent contracting literature acknowledges that there are unobservable quality dimensions (see, e.g., Kaarbøe and Siciliani, 2011) and we concentrate on those. Third, measuring quality is a complicated endeavor and creates problems in its own right (as a response to report cards Dranove et al. (2003), for instance, find selection behavior on the side of providers).
} 


$$
\begin{aligned}
& \frac{\partial W^{T}\left(T_{i j}, \gamma\right)}{\partial T_{i j}}=-\theta_{i j} \Psi_{i j}^{\prime}+\mu \theta_{i j}=0 \quad \Rightarrow \quad \Psi_{i j}^{\prime}=\mu \quad \forall i j \\
& \frac{\partial W^{T}\left(T_{i j}, \gamma\right)}{\partial \gamma}=\left(-c_{q}+[1+\alpha \beta] b_{q}\right) q_{\gamma}-\left(c_{e}+v_{e}\right) e_{\gamma}=0 .
\end{aligned}
$$

Like in the first-best solution individualized lump-sum transfers should be chosen such that marginal utilities are equalized. Equation 27) yields the optimal degree of cost sharing, $\gamma^{T}$, which, using the HCP's first order conditions (5) and (6), dictates $q^{T}$ and $e^{T}$ : :

$$
\alpha b_{q}\left(q^{T}\right)=\left(1-\gamma^{T}\right) c_{q}\left(q^{T}\right) \text { and } \quad-\left(1-\gamma^{T}\right) c_{e}\left(e^{T}\right)=v_{e}\left(e^{T}\right)
$$

Implementation of the first-best allocation is generally impossible as the HA has only one instrument, the cost sharing parameter $\gamma$, but two margins, namely, $q$ and $e$. It can easily be verified that a cost-sharing parameter $\gamma=0$ implements the efficient effort level as the provider is the residual claimant for his cost savings. Quality provision, however, will then be inefficient unless $\alpha(1-\beta)=1$. As $\alpha \in[0,1]$ and $\beta \in\{0,1\}$ first-best quality requires perfect altruism $\alpha=1$ and $\beta=0$ when $\gamma=0$. If $\alpha(1-\beta) \neq 1$ we get $\gamma \neq 0$ and with it a distortion of the HCP's incentives to exert effort. The HA can, thus, either implement the first-best cost reducing effort or the first-best level of quality but not both. The optimal cost sharing parameter, $\gamma^{T}$, optimally trades off the inefficiencies along the two dimensions.

\subsection{Third-Best-The Optimal Financing and Funding Interaction}

In addition to the resource constraints and the non-contractibility of quality and effort the HA now faces financing constraints. When optimal income taxation is no longer feasible the HA has to resort to alternative financing regimes, proportional income taxation and insurance premiums being the most prominent arrangements. We investigate the resulting third-best allocations in turn.

\subsubsection{Proportional Income Taxes}

With proportional income taxes the policy instruments are given by the tax rate $t$ and the cost sharing parameter $\gamma$. The optimization problem reads as

$$
\begin{array}{ll}
\max _{t, \gamma} & W^{t}(t, \tau, \gamma)=\sum_{i j} \theta_{i j} \Psi\left((1-t) y_{i}+\pi_{j}[b(q)-L]\right) \\
\text { s.t. } & \text { (5), (6) and (14). }
\end{array}
$$


Inserting the constraints, the optimization problem simplifies to

$$
W^{t}(\gamma)=\sum_{i j} \theta_{i j} \Psi\left(\left(1-\frac{\bar{\pi}}{\bar{y}}\left[c(q(\gamma), e(\gamma))+v(e(\gamma))-\alpha \beta b(q(\gamma))+\frac{F}{\bar{\pi}}\right]\right) y_{i}+\pi_{j}[b(q(\gamma))-L]\right)
$$

The optimal cost-sharing parameter, $\gamma^{t}$, is implicitly determined by the following first order condition

$$
\frac{\mathrm{d} W^{t}(\gamma)}{\mathrm{d} \gamma}=\sum_{i j} \theta_{i j} \Psi_{i j}^{\prime}\left(-\frac{\bar{\pi}}{\bar{y}} y_{i}\left[\left(c_{q}-\alpha \beta b_{q}\right) q_{\gamma}+\left(c_{e}+v_{e}\right) e_{\gamma}\right]+\pi_{j} b_{q} q_{\gamma}\right)=0
$$

where $q_{\gamma}$ and $e_{\gamma}$ are defined as in equations (7) and (8). To gain a better understanding of how redistributive concerns affect optimal cost-sharing under proportional income taxation $\gamma^{t}$ and thus optimal quality $q^{t}$ and effort $e^{t}$, we introduce the distributional characteristics of health risk and income (see Feldstein, 1972):

$$
\begin{gathered}
\xi^{\pi} \equiv \frac{\sum_{i j} \theta_{i j} \Psi_{i j}^{\prime} \pi_{j}-\sum_{i j} \theta_{i j} \Psi_{i j}^{\prime} \sum_{i j} \theta_{i j} \pi_{j}}{\sum_{i j} \theta_{i j} \Psi_{i j}^{\prime} \sum_{i j} \theta_{i j} \pi_{j}}, \\
\xi^{y} \equiv \frac{\sum_{i j} \theta_{i j} \Psi_{i j}^{\prime} y_{i}-\sum_{i j} \theta_{i j} \Psi_{i j}^{\prime} \sum_{i j} \theta_{i j} y_{i}}{\sum_{i j} \theta_{i j} \Psi_{i j}^{\prime} \sum_{i j} \theta_{i j} y_{i}} .
\end{gathered}
$$

These measures amount to the standardized covariances between the welfare weights the government attaches to a particular $i j$-type and health risk and income, respectively. For a utilitarian $\mathrm{HA}, \Psi^{\prime \prime}=0$, all individuals have the same welfare weight irrespective of health and income, that is, $\Psi_{i j}^{\prime}=\bar{\Psi}>0 \forall i j$. The absence of redistributive concerns is reflected in the distributional characteristics both assuming the value zero, $\xi^{\pi}=\xi^{y}=0$. Similarly, without agent heterogeneity the distributional characteristics would be zero: $\pi_{l}=\pi_{h}$ implies $\xi^{\pi}=0$ and $y_{p}=y_{r}$ implies $\xi^{y}=0$.

Using equations (31) and (32), equation (30) can be rewritten as

$$
\left(-c_{q}+[\Xi+\alpha \beta] b_{q}\right) q_{\gamma}-\left(v_{e}+c_{e}\right) e_{\gamma}=0
$$

where $\Xi \equiv\left(1+\xi^{\pi}\right) /\left(1+\xi^{y}\right)$. The following proposition states how the third-best allocation with proportional income taxes relates to the second-best outcome.

\section{Proposition 1 (Optimal policy with proportional income taxes)}

(i) $\xi^{\pi} \geq \xi^{y} \Leftrightarrow \gamma^{t} \geq \gamma^{T} \quad \Leftrightarrow \quad q^{t} \geq q^{T} \quad$ and $\quad e^{t} \leq e^{T}$.

(ii) The third-best allocation with proportional income taxes is identical to the second-best allocation if and only if $\xi^{y}=\xi^{\pi}=0$. 
The intuition for part $(i)$ is as follows 10 Whenever $\Xi>1$, which is equivalent to $\xi^{\pi}>\xi^{y}$, the marginal benefit of quality provision is higher under proportional income taxes than under optimal income taxation. The reason being the distributional consequences of improvements in quality. High risk individuals are more likely to benefit from higher quality care but, ceteris paribus, do not pay more taxes than low risk individuals. Additionally, rich individuals contribute more to the financing of better quality without benefitting more than poor individuals. The increase in quality, thus, implies more distribution towards poor and high risk individuals. These redistributive benefits have to be weighed against the additional distortion of effort incentives and the third-best optimal degree of cost sharing does so optimally. For $\Xi<1$ cost-sharing in the third-best is less pronounced than in the second-best and quality and effort levels compare accordingly. Optimal cost sharing in the third-best and second-best optima is identical if and only if $\Xi=1$. If $\xi^{\pi}=\xi^{y} \neq 0$, then health care provision is second-best efficient. As distributional concerns matter, however, the allocation is not second-best efficient. As a consequence of suboptimal health care financing the income redistribution is inefficient. Only in the absence of redistributive concerns, $\Psi^{\prime \prime}=0$, or when there is no need for redistribution, $\pi_{l}=\pi_{h}$ and $y_{p}=y_{r}$, the third-best and second-best allocations coincide (ii).

One can certainly speculate about how the distributional characteristics compare to one another. We conjecture that the HA attaches a higher welfare weight to the disadvantaged, that is, to individuals with high health risks, $\operatorname{Cov}\left(\Psi_{i j}^{\prime}, \pi_{j}\right)>0$, and low income, $\operatorname{Cov}\left(\Psi_{i j}^{\prime}, y_{i}\right)<0$. This is equivalent to $\xi^{\pi}>0$ and $\xi^{y}<0$ which implies $\Xi>1$ and with it more cost sharing, higher quality and less effort under proportional income taxation as compared to optimal income taxation.

\subsubsection{Insurance Premiums}

With insurance premiums the optimization problem of the HA is given by

$$
\begin{array}{ll}
\max _{p, \gamma} & W^{p}(p, \tau, \gamma)=\sum_{i j} \theta_{i j} \Psi\left(y_{i}-\left[(1-\varphi) \pi_{j}+\varphi \bar{\pi}\right] p+\pi_{j}[b(q)-L]\right) \\
\text { s.t. } & \text { (5), (6) and 14). }
\end{array}
$$

Employing the same approach as in the previous section and using equation (31), the first order condition for the optimal cost-sharing parameter $\gamma^{p}$ can be written as

$$
\left(-c_{q}+\left[\frac{1+\xi^{\pi}}{1+(1-\varphi) \xi^{\pi}}+\alpha \beta\right] b_{q}\right) q_{\gamma}-\left(c_{e}+v_{e}\right) e_{\gamma}=0 .
$$

\footnotetext{
${ }^{10}$ Analytically, the result follows - like in all other propositions and corollaries - from a simple comparison of the coefficients on quality in the respective first order conditions, here equations (27) and (33).
} 
This condition allows us to state our next proposition where we compare the results with insurance premiums to the second-best allocation.

\section{Proposition 2 (Optimal policy with insurance premiums)}

(i) When insurance premiums entail some redistribution, $\varphi>0$, then: $\xi^{\pi} \geq 0 \quad \Leftrightarrow \quad \gamma^{p} \geq \gamma^{T} \quad \Leftrightarrow \quad q^{p} \geq q^{T} \quad$ and $\quad e^{p} \leq e^{T}$.

(ii) For purely risk-based premiums, $\varphi=0$, health care provision is second-best efficient while health care financing is not.

(iii) The third-best allocation with insurance premiums is identical to the second-best allocation if and only if $\xi^{y}=\xi^{\pi}=0$.

The intuition is similar to the one of Proposition 1. Suppose that insurance premiums imply some redistribution from low-risk to high-risk individuals as stated in part (i) of the proposition. Then an increase in quality triggered by more cost sharing benefits high-risk individuals with a higher probability than low-risk individuals. The resulting increase in insurance premiums, however, is not shared accordingly when premiums are partially pooled. High-risk individuals disproportionately benefit from an increase in quality making such improvements desirable whenever the HA attaches a higher welfare weight to high-risk individuals than to low-risk individuals, i.e., whenever $\xi^{\pi}>0$. In this case it pays off to distort quality and effort away from its second-best levels as the resulting improvement in the distribution of income outweighs the associated losses in efficiency. There is no such distributional benefit when premiums are purely risk-rated, (ii). This result carries an important policy message: second-best optimal health care provision can be achieved by both financing regimes, optimal income taxation and risk-rated insurance premiums. It emphasizes that an isolated (second-best) analysis of provider payment rests on strong assumptions regarding the financing of health care - unless, (iii), the HA has no redistributive concerns.

Combining Propositions 1 and 2 we find that the optimal policy is sensitive to the mode of health care financing when redistributive concerns matter. Only in the special case of no redistributive concerns is the redistribution of income, implied by a change from one health care financing regime to the other, welfare neutral. We emphasize this in the following corollary.

\section{Corollary 1 (Optimal policy: proportional income taxes vs. insurance premiums)} $(1-\varphi) \xi^{\pi} \geq \xi^{y} \quad \Leftrightarrow \quad \gamma^{t} \geq \gamma^{p} \quad \Leftrightarrow \quad q^{t} \geq q^{p} \quad$ and $\quad e^{t} \leq e^{p}$.

In general, the comparison of cost sharing across financing regimes depends on the welfare weights the HA attaches to the different types. We already suggested above, that the HA may wish to redistribute towards the disadvantaged in the society, namely, to high-risk low-income agents. This implies $\xi^{\pi}>0$ and $\xi^{y}<0$ and, following the corollary, less cost-sharing with 
insurance premiums independent of the extent of pooling $\varphi$. As a result, health care quality in tax based systems is expected to exceed quality in insurance financed systems. It is the other way round for cost reducing effort, leading to higher expected health care expenses in the former systems than in the latter.

\section{Political Implementability of Optimal Policies}

In the previous section we assumed a normative perspective and investigated the optimal health care policies under optimal income taxation (first-best and second-best) and contrasted them with alternative financing regimes (third-best). Implementing an optimal cost sharing parameter, however, may not be feasible politically. Under majority voting, policy makers will commit to policies that maximize the number of votes rather than the social objective. Taking a median voter approach, this section derives the political equilibria under proportional income taxation and insurance premiums and contrasts them with the respective third-best and second-best policies.

With a continuum of individuals each voting agent has zero mass, so that no individual vote can change the outcome of the election. We let all agents alive cast a ballot over the cost-sharing parameter $\gamma$. Although it appears more natural to let voters decide on the parameter which determines the financing of health care rather than on one parameter of the reimbursement scheme, this approach is without loss of generality. Remember that in an economic equilibrium only one of the three policy instruments can be set freely. The other two are then residually determined by the HCP's participation constraint and the HA's budget constraint. It is thus irrelevant whether voters decide on $\gamma, t / p$ or $\tau$. We opt for the former as it allows for a direct comparison of the equilibrium allocations of the two approaches, normative and positive.

\subsection{Proportional Income Taxes}

Individuals maximize their indirect utility function (18) with respect to the cost-sharing parameter $\gamma$ subject to the constraint on the tax rate $t$ given by equation (14). The first order condition of an $i j$-type amounts td

$$
\frac{\mathrm{d} V_{i j}^{t}(\gamma)}{\mathrm{d} \gamma}=\left(-\delta_{i} c_{q}+\left[\delta_{j}+\alpha \beta \delta_{i}\right] b_{q}\right) q_{\gamma}-\delta_{i}\left(c_{e}+v_{e}\right) e_{\gamma}=0
$$

\footnotetext{
${ }^{11}$ For convenience we neglect the constraint $\gamma \in[0,1]$. Both, the ordering of types with respect to their most preferred cost sharing parameters as well as the comparison of allocations, normative and positive, remain valid.
} 
where we defined $\delta_{i} \equiv y_{i} / \bar{y}$ and $\delta_{j} \equiv \pi_{j} / \bar{\pi}$. The above equation implicitly determines the most preferred cost-sharing parameter $\gamma_{i j}^{t}$ of a type-ij individual. ${ }^{12}$ To see how the most preferred cost-sharing parameter depends on income and risk we apply the implicit function theorem which, observing the first order conditions of the HCP, equations (5) and (6), yields

$$
\begin{aligned}
\frac{\mathrm{d} \gamma_{i j}^{t}}{\mathrm{~d} \delta_{i}} & =\frac{\left(\gamma c_{q}+\alpha(1-\beta) b_{q}\right) q_{\gamma}+c_{e} e_{\gamma}}{S O C_{i j}}<0 \quad \text { and } \\
\frac{\mathrm{d} \gamma_{i j}^{t}}{\mathrm{~d} \delta_{j}} & =-\frac{b_{q} q_{\gamma}}{S O C_{i j}}>0 .
\end{aligned}
$$

These inequalities allow us to order the four types according to their most preferred cost-sharing parameter and with it to identify the median voter.

\section{Lemma 1 (Median voter: proportional income taxes)}

The most preferred cost-sharing parameters can be ordered as follows: $\gamma_{r l}^{t}<\min \left\{\gamma_{p l}^{t}, \gamma_{r h}^{t}\right\} \leq$ $\max \left\{\gamma_{p l}^{t}, \gamma_{r h}^{t}\right\}<\gamma_{p h}^{t}$. The median voter is a pl-type agent, i.e., $\gamma_{m}^{t}=\gamma_{p l}^{t}$.

Equation (37) implies $\gamma_{r j}^{t}<\gamma_{p j}^{t}$. For a given risk type high income agents contribute more to the financing of the health care scheme than low income agents. As the risk type is given, the benefit from more cost sharing in terms of higher quality is uniform across income types making cost sharing less attractive for high income individuals. Similarly, equation (38) implies $\gamma_{i l}^{t}<\gamma_{i h}^{t}$. For a given income, the expected benefits from quality improvements triggered by more cost-sharing are higher for high-risk individuals than for low-risk individuals. As financing costs are uniform across risk types, high-risk agents prefer more cost-sharing than low risk agents. Although the comparison of the most preferred cost sharing parameters of $p l$-types and $r h$-types generally depends on the distribution of types, identification of the median voter is straightforward: as neither the high-risks nor the rich can form a majority $\left(\theta_{h}<0.5\right.$ and $\theta_{r}<0.5$, respectively), the median voter is a type- $p l$ agent who either forms a majority with low-income high-risk individuals or with high-income low-risk individuals.

Inserting the median voter's type into equation (36) and dividing by $\delta_{p}$ allows a direct comparison with the third-best policy under proportional income taxation.

$$
\left(-c_{q}+[\Delta+\alpha \beta] b_{q}\right) q_{\gamma}-\left(c_{e}+v_{e}\right) e_{\gamma}=0
$$

where $\Delta \equiv \delta_{l} / \delta_{p}$. While $\delta_{l} \leq 1$ and $\delta_{p} \leq 1$ measure the inequity in health and income respectively, their fraction, $\Delta$, is a measure of the relative inequity between the two dimensions.

\footnotetext{
${ }^{12}$ In the following, we assume that the second order condition is negative, $\mathrm{d}^{2} V_{i j}^{t} / \mathrm{d} \gamma^{2} \equiv S O C_{i j}<0$, implying that preferences are single-peaked (see Appendix A.1.2.
} 


\section{Proposition 3 (Political outcome with proportional income taxes)}

(i) The relationship between cost sharing arrangements in the third-best optimum and the political equilibrium depends on how the relative inequity along income and risk relates to the distributional characteristics of cost sharing with respect to these two dimensions. More precisely, we have: $\Xi \gtreqless \Delta \Leftrightarrow \gamma^{t} \gtreqless \gamma_{m}^{t} \quad \Leftrightarrow \quad q^{t} \gtreqless q_{m}^{t} \quad$ and $\quad e^{t} \lesseqgtr e_{m}^{t}$.

(ii) Health care provision is second-best efficient if and only if $\Delta=1$. Second-best efficiency of the allocation requires the absence of redistributive concerns, $\xi^{\pi}=\xi^{y}=0$.

A comparison to the first order condition for the cost-sharing parameter in the third-best, equation (33), shows that the difference between these two equations depends on $\Delta$ and $\Xi$. We discuss the three possible cases in turn, (i). $\Delta<\Xi$ : the benefits of the quality of care receive a lower weight in the median voter's first order condition than in the HA's first order condition. Since quality provision can be stimulated by cost sharing, $q_{\gamma}>0$, the cost sharing parameter will be larger in the normative equilibrium than in the political one ${ }^{13}$ Suppose there is no inequity in risk, then $\Delta<\Xi \Leftrightarrow \delta_{p}>1+\xi^{y}$. This condition can only be satisfied if the HA aims at redistributing towards the poor, that is, if $\xi^{y}<0$. The smaller $\xi^{y}$ the more pronounced are the redistributive concerns of the HA. If the inequality in income is sufficiently small as compared to the distributional preferences of the HA, $\delta_{p}>1+\xi^{y}$, so are the benefits accruing to the median voter from improvements in quality. This, in turn, implies less cost sharing in the political outcome than in the optimal one. Similarly, suppose there is no inequity in income, then $\Delta<\Xi \Leftrightarrow \delta_{l}<1+\xi^{\pi}$. High-risk individuals disproportionately benefit from quality improvements. As the median voter is a low-risk agent, he aims at limiting the redistribution towards high-risk individuals and he does so by implementing a low cost sharing rate. As the distributional preferences of the HA will usually point in the opposite direction, $\xi^{\pi}>0$, cost sharing is more pronounced in the third-best allocation than in the political outcome. These arguments extend to the case with inequities in both, income and risk. $\Delta>\Xi$ : the cost sharing benefit accruing to the median voter is larger than in the social objective as, due to the sufficiently low health risk inequality as compared to income inequality, the benefits of better quality care are distributed more evenly. In that sense the redistribution is better targeted towards the median voter. The HA's redistributive concerns are lower so that her cost sharing incentives are weaker. $\Delta=\Xi$ : although this is a knife-edge case it is noteworthy that the distributional preferences of the median voter and the HA are aligned so that the political outcome is identical to the third-best allocation.

\footnotetext{
${ }^{13}$ To see this formally observe
}

$$
\frac{\mathrm{d} \gamma_{m}^{t}}{\mathrm{~d} \Delta}=-\frac{b_{q} q_{\gamma}}{S O C_{i j}}>0
$$


If the inequality in health risk and income is identical, $\Delta=1$, then the political outcome with health care financing through proportional income taxation leads to second-best efficient health care provision, (ii). The reason is that the median voter's benefits from cost sharing through improvements in the distribution of income are exactly compensated by the worsening of the distribution of health benefits. Finally, this allocation is second-best efficient if $\xi^{\pi}=\xi^{y}=0$.

We argued above that the HA likely attaches a higher welfare weight to high-risk or lowincome individuals than to low risk or high income ones. But then $\Xi>1$ and the third-best can only be implemented through a majority voting process if the inequality in health risk is smaller than the inequality in income such that $\Xi=\Delta>1$. In general, however, the second-best cannot be achieved as a political equilibrium.

\subsection{Insurance Premiums}

When health care financing is through insurance premiums, individual preferences over the cost sharing parameter are given by equation (19). The corresponding first order condition for $\gamma$ is given by

$$
\begin{array}{r}
\frac{\mathrm{d} V_{i j}^{p}(\gamma)}{\mathrm{d} \gamma}=-\left[(1-\varphi) \pi_{j}+\varphi \bar{\pi}\right]\left[c_{q} q_{\gamma}+c_{e} e_{\gamma}+v_{e} e_{\gamma}-\alpha \beta b_{q} q_{\gamma}\right]+\pi_{j} b_{q} q_{\gamma}=0 \\
\Leftrightarrow\left(-c_{q}+\left[\frac{\pi_{j}}{(1-\varphi) \pi_{j}+\varphi \bar{\pi}}+\alpha \beta\right] b_{q}\right) q_{\gamma}-\left(c_{e}+v_{e}\right) e_{\gamma}=0
\end{array}
$$

First, we note that for purely risk-based premiums, $\varphi=0$, all individuals have identical most preferred cost sharing rates so that there is no conflict in the electorate on how to shape health care funding. If premiums include a redistributive element, $\varphi>0$, then high-risk individuals benefit more from health care provision and vote for a higher cost-sharing parameter than lowrisk agents: $\mathrm{d} \gamma_{i j}^{p} / \mathrm{d} \pi_{j}>0$. This allows us to order the four types by their most preferred cost sharing parameter and, as a result, to identify the median voter.

\section{Lemma 2 (Median voter: insurance premiums)}

The most preferred cost-sharing parameters can be ordered as follows: $\gamma_{r l}^{p}=\gamma_{p l}^{p} \leq \gamma_{r h}^{p}=\gamma_{p h}^{p}$. The median voter is an l-type agent, i.e., $\gamma_{m}^{p}=\gamma_{i l}^{p}$ for $i=r, p$.

As rich low-risk individuals share their cost sharing preferences with poor low-risk individuals there will always be a majority for their most preferred cost sharing parameter. Inserting $\pi_{l}$ into equation 40 we arrive at our next proposition.

\section{Proposition 4 (Political outcome with insurance premiums)}

(i) When insurance premiums entail some redistribution, $\varphi>0$, then the relationship between cost sharing arrangements in the third-best optimum and the political equilibrium depends 
on how the inequity in risk relates to the distributional characteristic of risk. More precisely, we have: $1+\xi^{\pi} \geq \delta_{l} \Leftrightarrow \gamma^{p} \geq \gamma_{m}^{p} \Leftrightarrow q^{p} \geq q_{m}^{p}$ and $e^{p} \leq e_{m}^{p}$.

(ii) Health care provision is second-best efficient if and only if $\varphi=0$. Second-best efficiency of the allocation requires the absence of redistributive concerns, $\xi^{\pi}=\xi^{y}=0$.

If the HA aims at redistributing towards high-risk individuals, $\xi^{\pi}>0$, then the condition formulated in part (i) of the proposition always holds. High cost sharing parameters imply generous redistribution towards high risk individuals. Other than the HA the median voter, a low-risk agent, dislikes redistribution towards high-risk types. Accordingly, cost sharing is less pronounced in the political outcome than under the third-best policy. This ordering can only be reversed if the HA has a sufficiently strong preference to redistribute towards low-risk type agents. (ii), and rather remarkably, for purely risk-based premiums, $\varphi=0$, the incentives of voters are aligned with those of the HA in both, the second-best and the third-best environment; equations (27), (35) and (40) coincide. Although this result appears somewhat surprising, the intuition is relatively straightforward. The optimal allocations trade-off inefficiencies along cost reduction effort and quality and this trade-off is not blurred by redistributive motives. Similarly, in the political game redistribution plays no role as risk-based premiums preclude any form of redistribution. The third-best allocation can thus be implemented through a majority voting process. In contrast to the proportional income taxation case, this result holds independent of agent heterogeneity. Finally, note that for purely risk-based premiums health care provision is second-best efficient. The resulting income distribution may still be inefficient unless there are no redistributive concerns.

Combining Propositions 3 and 4 we arrive at the following corollary that facilitates comparison of political outcomes under alternative financing regimes.

Corollary 2 (Political outcome: proportional income taxes vs. insurance premiums) $\delta_{p}-\delta_{l} \leq \varphi\left(1-\delta_{l}\right) \quad \Leftrightarrow \quad \gamma_{m}^{t} \geq \gamma_{m}^{p} \Leftrightarrow q_{m}^{t} \geq q_{m}^{p} \quad$ and $\quad e_{m}^{t} \leq e_{m}^{p}$.

The comparison of cost sharing across financing regimes hinges on how risk pooling, as measured by $\varphi$, relates to the relative inequity between income and risk. Only in the knife-edge case, $\delta_{p}-\delta_{l}=\varphi\left(1-\delta_{l}\right)$, is health care provision under majority voting invariant to a change from insurance premiums to proportional income taxes.

\section{Conclusion}

Usually health care financing and health care funding are studied in isolation. This paper simultaneously analyzed the financing of health care and provider payment and thereby filled 
a gap in the literature. Considering a multi-task agency framework we derived the optimal allocations under varying constraints. These included alternate financing regimes yielding the first-best, second-best and third-best allocations. We then investigated whether the optimal policies can be implemented as political equilibria under majority voting. A pairwise comparison of allocations allowed us to identify conditions under which allocations coincide. In other words, we showed when the mode of health care financing is irrelevant and when optimal policies can be implemented as a median voter equilibrium (Figure 1 summarizes the respective conditions).

More precisely, when the quality of care and cost-reducing effort are non-contractible but optimal income taxation is feasible, the optimal allocation is second-best efficient. While the income distribution is optimal, the inefficiencies along quality and effort are, using supply-side cost-sharing, optimally traded off against one another. If optimal income taxation is not feasible and if the health authority has redistributive concerns, health care financing has redistributive consequences which, in turn, will affect optimal provider payment.

With proportional income taxation health care financing implies a redistribution from highincome to low-income individuals and from low-risk to high-risk individuals. The extent to which the health authority distorts provider payment away from its second-best (and with it health care provision) depends on individual heterogeneity in risk and income and on the welfare weights the health authority attaches to the different types. We show that Feldstein's (1972) distributional characteristics can be used to characterize the third-best allocation. If the distributional characteristics of income and risk are identical, so are second-best and third-best health care provision. As income distributions might differ, the third-best allocation is not second-best efficient unless both distributional characteristics are nil. This is the case when the health authority has no redistributive concerns, or in the absence of individual heterogeneity. The political outcome is governed by the preferences of the median voter who happens to be a poor, low-risk agent. With proportional income taxation the median voter contributes less to the financing of the health care scheme than the average voter but at the same time needs health care with a lower probability than the average voter. The median voter's preferences for cost-sharing are thus driven by the relative inequity between risk and income. These preferences will likely differ from the ones of the health authority so that the third-best allocation can generally not be implemented as a median voter equilibrium. Figure 1 below gives the condition under which the two are identical. It should be noted that the political outcome may yield second-best health care provision, which is the case whenever the inequality in risk is identical to the inequality in income. Only in the absence of individual heterogeneity or redistributive concerns is this allocation second-best efficient.

When considering insurance premiums as a mode of health care financing, redistributive consequences are driven by the extent of risk pooling. In the extreme case of risk-based premiums 
(no pooling) the third-best allocation yields second-best health care provision. The reason is that risk-based premiums preclude any form of redistribution so that the health authority refrains from distorting health care provision away from its second-best. But this also implies that there is no conflict in the electorate how to shape health care provision. The third-best allocation can thus be implemented as a political equilibrium and health care provision is second-best efficient. As the income distribution might not be optimal the resulting allocations will generally not be second-best efficient. In the event of (partially) pooled premiums, health care financing through insurance premiums involves a redistributive element. Income is redistributed from low-risk to high-risk individuals. Redistribution across income types is ruled out. Consequently, the thirdbest allocation and the political outcome will depend on the distributional characteristic of risk and the inequity in risk, respectively. Only in the event of aligned cost-sharing preferences of the health authority and the median voter can the third-best allocation be implemented by majority voting. Again, the respective condition is given in Figure 1.

Finally, we derived conditions under which health care financing is irrelevant in third-best and political economy environments. It turns out that health care financing will generally matter unless risk pooling relates to the distributional characteristics and individual heterogeneity in the way laid out in Figure 1 below.

The framework we adopted only considers public health care and abstracts from additional public programs like, most importantly, an income transfer scheme. An immediate implication is that the former program implicitly assumes the redistributive role of the latter program (Cremer and Gahvari, 1997). Our results are largely robust to adding an income transfer scheme in the sense that individual heterogeneity, redistributive preferences, and health financing would still matter. The reason is that, apart from risk-based premiums, health care financing is inherently redistributive. Only in rather extreme and unrealistic cases would the size of the public health care program be invariant to health care financing and be independent of individual heterogeneity and redistributive preferences. More precisely, if no additional redistribution over and above the redistribution via the transfer scheme was beneficial (the transfer scheme is financed by optimal income taxes $\left.{ }^{14}\right)$ or if the public health care program and the transfer scheme have identical redistributive properties (e.g., if both programs are financed by proportional income taxes and transfers within the income scheme are perfectly correlated with risk).

Our results make very clear that an isolated analysis of provider payment rests on very strong assumptions regarding the mode of health care financing (optimal income taxation or risk-based premiums) or the redistributive concerns of the health authority (none). This has a bearing on the applicability of the results derived in the optimal provider payment literature. The qualitycost containment tradeoff will generally differ internationally. In principle, our framework could

\footnotetext{
${ }^{14}$ This is, of course, equivalent to assuming health care financing through optimal income taxes.
} 
be used to derive testable hypothesis regarding the relationship between health care financing and supply-side cost-sharing. Actual hypothesis testing would require accurate data on the degree of cost-sharing, Feldstein's distributional characteristics, and individual heterogeneity. To the best of our knowledge, such data is currently not available so that we leave the empirical analysis for future research.

\section{A Appendix}

\section{A.1 Second-Order Conditions}

\section{A.1.1 The Health Care Provider}

Since the two leading principal minors alternate in sign:

$$
\begin{aligned}
& \left|H_{q q}\right|=\left|\alpha b_{q q}-(1-\gamma) c_{q q}\right|<0 \\
& \left|\begin{array}{ll}
H_{q q} & H_{q e} \\
H_{e q} & H_{e e}
\end{array}\right|=\left|\begin{array}{cc}
\alpha b_{q q}-(1-\gamma) c_{q q} & 0 \\
0 & (1-\gamma) c_{e e}+v_{e e}
\end{array}\right|>0
\end{aligned}
$$

due to $b_{q q}<0, c_{q q}>0, c_{e e} \geq 0$ and $v_{e e}>0$, the HCP's chosen quality level, $q(\gamma ; \alpha)$, and cost-reducing effort, $e(\gamma ; \alpha)$, are a maximum of the objective function $H(q, e)$.

\section{A.1.2 Individuals}

The second-order condition of individual- $i j$ amounts to

$$
\begin{aligned}
\frac{\mathrm{d}^{2} V_{i j}^{t}(\gamma)}{\mathrm{d} \gamma^{2}}= & \underbrace{\left(-\delta_{i} c_{q q}+\left(\delta_{j}+\alpha \beta \delta_{i}\right) b_{q q}\right)\left(q_{\gamma}\right)^{2}}_{<0}+\underbrace{\left(-\delta_{i} c_{q}+\left(\delta_{j}+\alpha \beta \delta_{i}\right) b_{q}\right) q_{\gamma \gamma}}_{\gtrless 0} \\
& \underbrace{-\delta_{i}\left(c_{e e}+v_{e e}\right)\left(e_{\gamma}\right)^{2}}_{<0} \underbrace{-\delta_{i}\left(c_{e}+v_{e}\right) e_{\gamma \gamma}}_{<0} .
\end{aligned}
$$

\section{A.2 Comparative Statics}

Taking the total derivative of the HCP's first order conditions (6) and (5) yields

$$
\begin{aligned}
\left(\alpha b_{q q}-(1-\gamma) c_{q q}\right) \mathrm{d} q & =-c_{q} \mathrm{~d} \gamma-b_{q} \mathrm{~d} \alpha \\
\left((1-\gamma) c_{e e}+v_{e e}\right) \mathrm{d} e & =c_{e} \mathrm{~d} \gamma
\end{aligned}
$$

as we assume $c_{q e}=0$. The above system of equations can be written as

$$
\left[\begin{array}{l}
\mathrm{d} q \\
\mathrm{~d} e
\end{array}\right]=\frac{1}{D}\left[\begin{array}{cc}
(1-\gamma) c_{e e}+v_{e e} & 0 \\
0 & \alpha b_{q q}-(1-\gamma) c_{q q}
\end{array}\right]\left[\begin{array}{c}
-c_{q} \mathrm{~d} \gamma-b_{q} \mathrm{~d} \alpha \\
c_{e} \mathrm{~d} \gamma
\end{array}\right]
$$


where $D=\left(\alpha b_{q q}-(1-\gamma) c_{q q}\right)\left((1-\gamma) c_{e e}+v_{e e}\right)>0$. Hence, we have

$$
\begin{aligned}
& \frac{\mathrm{d} q}{\mathrm{~d} \gamma}=\frac{-\left((1-\gamma) c_{e e}+v_{e e}\right) c_{q}}{D}=\frac{-c_{q}}{\alpha b_{q q}-(1-\gamma) c_{q q}}>0 \\
& \frac{\mathrm{d} e}{\mathrm{~d} \gamma}=\frac{\left(\alpha b_{q q}-(1-\gamma) c_{q q}\right) c_{e}}{D}=\frac{c_{e}}{(1-\gamma) c_{e e}+v_{e e}}<0 .
\end{aligned}
$$

Additionally, we have

$$
\begin{aligned}
\frac{\mathrm{d}^{2} q}{\mathrm{~d} \gamma^{2}} & =\frac{c_{q} c_{q q}}{\left(\alpha b_{q q}-(1-\gamma) c_{q q}\right)^{2}}>0 \\
\frac{\mathrm{d}^{2} e}{\mathrm{~d} \gamma^{2}} & =\frac{c_{e} c_{e e}}{\left((1-\gamma) c_{e e}+v_{e e}\right)^{2}}<0
\end{aligned}
$$

\section{A.3 The Health Care Financing and Funding Interaction}

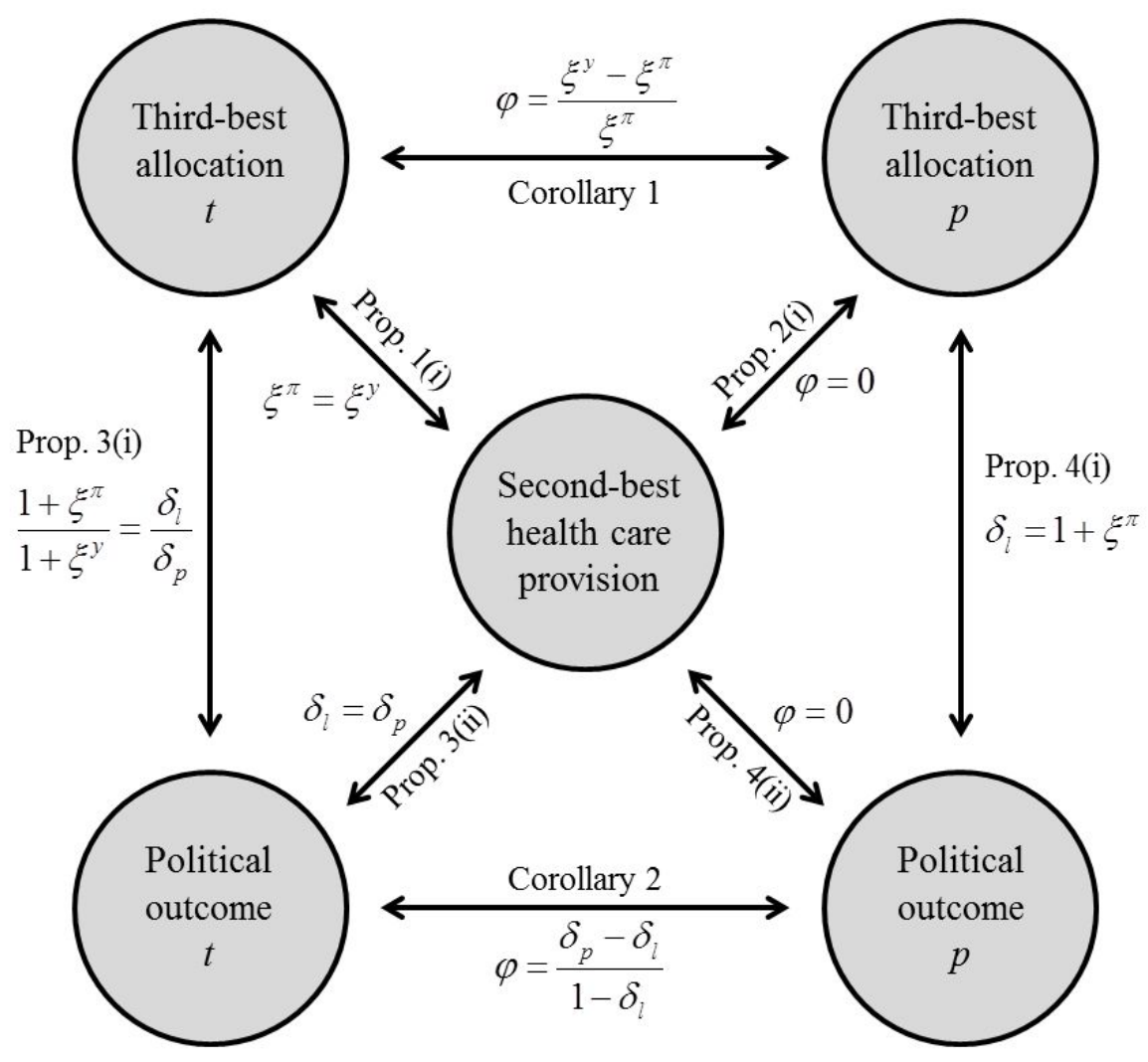

Figure 1: Allocations under optimal policies and their political implementation under majority voting. 


\section{References}

Chalkley, M. and J.M. Malcomson, "Contracting for Health Services with Unmonitored Quality," The Economic Journal, 1998a, 108, 1093-1110.

, "Contracting for Health Services when Patient Demand does not Reflect Quality," Journal of Health Economics, 1998b, 17, 1-19.

__ , "Government Purchasing of Health Services," Handbook of Health Economics, 2000, 1a, $847-890$.

Cremer, H. and F. Gahvari, "In-Kind Transfers, Self-Selection and Optimal Tax Policy," European Economic Review, 1997, 41, 97-114.

Dranove, D., Kessler, D., McClelland, M. and M. Satterthwaite, "Is More Information Better? The Effects of Report Cards on Health Care Providers," Journal of Political Economy, 2003, 111, 555-588.

Eggleston, K., "Multitasking and Mixed Systems for Provider Payment," Journal of Health Economics, 2005, 24, 211-223.

Ellis, R.P. and T.G. McGuire, "Optimal Payment Systems for Health Services," Journal of Health Economics, 1990, 9, 375-396.

Epple, D. and R.E. Romano, "Public Provision of Private Goods," Journal of Political Economy, 1996, 104, 57-84.

Feldstein, M.S., "Distributional Equity and the Optimal Structure of Public Prices," American Economic Review, 1972, 62, 32-36.

Gouveia, M., "Majority Rule and the Public Provision of a Private Good," Public Choice, 1997, 93, 221-244.

Jack, W., "Purchasing Health Care Services from Providers with Unknown Altruism," Journal of Health Economics, 2005, 24, 73-93.

Kaarbøe, O.M. and L. Siciliani, "Multitasking, Quality and Pay for Performance," Health Economics, 2011, 20, 225-238.

Kifmann, M., "Health Insurance in a Democracy: Why is It Public and Why are Premiums Income Related?," Public Choice, 2005, 124, 283-308.

Nuscheler, R. and K. Roeder, "The Political Economy of Long-Term Care," European Economic Review, 2013, 62, 154-173. 
Ma, C.A., "Health Care Payment Systems: Cost and Quality Incentives," Journal of Economics \& Management Strategy, 1994, 3, 93-112.

Ma, C.A. and T.G. McGuire, "Optimal Health Insurance and Provider Payment," American Economic Review, 1999, 89, 685-704.

Pauly, M.V. and B. Herring, "Risk Pooling and Regulation: Policy and Reality in Today's Individual Health Insurance Market," Health Affairs, 2007, 26, 770-779.

Zeckhauser, R., "Medical Insurance: A Case Study of the Tradeoff between Risk Spreading and Appropriate Incentives," Journal of Economic Theory, 1970, 2, 10-26. 